\title{
The prevalence of enteroviral capsid protein vp1 immunostaining in pancreatic islets in human type 1 diabetes
}

\author{
S. J. Richardson • A. Willcox • A. J. Bone • \\ A. K. Foulis • N. G. Morgan
}

Received: 4 September 2008 / Accepted: 9 January 2009/Published online: 6 March 2009

(C) Springer-Verlag 2009

\begin{abstract}
Aims/hypothesis Evidence that the beta cells of human patients with type 1 diabetes can be infected with enterovirus is accumulating, but it remains unclear whether such infections occur at high frequency and are important in the disease process. We have now assessed the prevalence of enteroviral capsid protein vp1 (vp1) staining in a large cohort of autopsy pancreases of recent-onset type 1 diabetic patients and a range of controls.

Methods Serial sections of paraffin-embedded pancreatic autopsy samples from 72 recent-onset type 1 diabetes patients and up to 161 controls were immunostained for insulin, glucagon, vp1, double-stranded RNA activated protein kinase R (PKR) and MHC class I.

Results vp1-immunopositive cells were detected in multiple islets of 44 out of 72 young recent-onset type 1 diabetic patients, compared with a total of only three islets in three out of 50 neonatal and paediatric normal controls. vp1
\end{abstract}

Electronic supplementary material The online version of this article (doi:10.1007/s00125-009-1276-0) contains supplementary material, which is available to authorised users.

S. J. Richardson · A. Willcox $\cdot$ N. G. Morgan $(\bowtie)$

Institute of Biomedical and Clinical Sciences,

Peninsula Medical School,

John Bull Building, Tamar Science Park,

Plymouth PL6 8BU, UK

e-mail: noel.morgan@pms.ac.uk

\section{A. J. Bone}

School of Pharmacy and Biomolecular Sciences,

University of Brighton,

Moulescoomb,

Brighton, UK

\section{A. K. Foulis}

Department of Pathology, Royal Infirmary,

Glasgow, UK staining was restricted to insulin-containing beta cells. Among the control pancreases, vpl immunopositivity was also observed in some islets from ten out of 25 type 2 diabetic patients. A strong correlation was established between islet cell vp1 positivity and PKR production in insulin-containing islets of both type 1 and type 2 diabetic patients, consistent with a persistent viral infection of the islets.

Conclusions/interpretation Immunoreactive vp1 is commonly found in the islets of recent-onset type 1 diabetes patients, but only rarely in normal paediatric controls. vp1 immunostaining was also observed in some islets of type 2 diabetes patients, suggesting that the phenomenon is not restricted to type 1 diabetes patients.

Keywords Beta cells $\cdot$ Enterovirus $\cdot$ Environmental triggers · Insulitis · Islets of Langerhans · MHC · PKR · Type 1 diabetes . Type 2 diabetes

$\begin{array}{ll}\text { Abbreviations } \\ \text { CVB } & \text { Coxsackie B enterovirus } \\ \text { ICI } & \text { Insulin-containing islet } \\ \text { IDI } & \text { Insulin-deficient islet } \\ \text { PKR } & \text { Double-stranded RNA activated protein kinase R } \\ \text { vp1 } & \text { Enteroviral capsid protein vp1 }\end{array}$

\section{Introduction}

Enteroviral infection has long been implicated in triggering autoimmunity, islet inflammation (insulitis) and beta cell death in human type 1 diabetes. Much of the evidence is circumstantial [1] but includes observations that antibodies against common Coxsackie B enteroviruses (CVB) and 
enteroviral RNA occur more frequently in the circulation of recent-onset type 1 diabetes patients than in controls [2-10].

More direct support for a viral aetiology has come from the demonstration that a strain of CVB4, isolated from the pancreas of a recent-onset type 1 diabetic patient, was capable of inducing diabetes in mice [11]. In addition, the presence of enteroviral RNA was revealed by in situ hybridisation in the islets of four recent-onset type 1 diabetes cases [12] and the enteroviral capsid protein vp1, was detected in the beta cells of two of five patients with type 1 diabetes, and in a sixth diabetic patient who had received a recent pancreatic transplant [13]. Electron microscopic analysis revealed the presence of virus particles within the islet beta cells of vp1-positive cases, and a strain of CVB4 was isolated from the pancreas of one patient. This was capable of infecting normal human islets in vitro, resulting in their functional impairment [13].

Enteroviral infection can result in the production of double-stranded RNA (dsRNA) within cells [14] and dsRNA-activated protein kinase R (PKR) was upregulated in islets infected by CVB5 in vitro [15]. Similarly, CVBinfected islets produced IFN- $\alpha$ in vitro [16] and this has been associated with hyperexpression of class I MHC [17].

In view of these findings, we considered it important to establish the prevalence of immunoreactive vp1 staining in the islets of a large cohort of recent-onset type 1 diabetes cases and in a wide range of relevant controls. We have also established whether an antiviral response is mounted in the islets of these patients by immunostaining for PKR. Finally, we have assessed whether vp1 staining occurs independently of insulitis.

\section{Methods}

Individuals Formalin-fixed, paraffin-embedded pancreatic autopsy tissue from 72 patients with recent-onset type 1 diabetes mellitus, whose pancreatic histology has been described previously [18] was used, with ethical permission. The cases were from various hospitals and had been fixed in buffered formalin, formol saline, formol saline with added mercuric chloride, or Bouin's fixative, with no standardisation of fixation time. The cohort consisted of 41 female and 31 male patients with a mean age of $12.65 \pm$ 1.1 years (range $1-42$ years) and a mean time since diagnosis of $8.2 \pm 4.1$ months (range 0-6 years). Control autopsy tissues, fixed in a similar variety of fixatives, were five culture-proven Coxsackie virus-infected neonatal pancreases and hearts; 11 normal neonatal pancreases ( $<1$ month); three normal neonatal hearts; 39 normal paediatric pancreases (age range 6 weeks-17 years); 11 pancreases from cystic fibrosis patients with a mean age of $8.1 \pm$ 1.7 years (age range 2-19 years); 69 normal adult pancreases and 25 pancreases from adult patients with type 2 diabetes.

Immunohistochemistry serial sections $(4 \mu \mathrm{m})$ were cut from each case and mounted on glass slides coated in (3-aminopropyl)-triethoxysilane (Sigma, Poole, Dorset, UK). Sections were processed and labelled using a standard immunoperoxidase technique for paraffin sections. With the exception of glucagon, insulin and MHC class I, all other antigens were unmasked by heat-induced epitope retrieval. Sections to be labelled with anti-PKR and anti-CD45 were heated in $10 \mathrm{mmol} / 1$ citrate buffer $\mathrm{pH} 6.0$, while those to be labelled with Dako anti-vp1 (5D8/1; Dako Cytomation, Ely, UK), polyclonal anti-vp1, or anti-pan-enterovirus (9D5; Millipore, Livingston, UK) were heated in $1 \mathrm{mmol} / \mathrm{l}$ EDTA, $\mathrm{pH}$ 8.0. See Electronic supplementary material (ESM) Table 1 for details of antibody dilutions and sources.

Primary antibodies were applied for $30 \mathrm{~min}$ at room temperature (except for anti-pan-enterovirus [9D5] and anti-PKR, which were incubated overnight at $4{ }^{\circ} \mathrm{C}$ ) and a Dako REAL Envision Detection System used for antigen detection. Some slides were processed in the absence of primary antibody or with isotype control antisera to confirm the specificity of labelling. Slides were analysed by light microscopy.

Immunofluorescence staining To determine the islet cell subtypes infected by enterovirus, double immunofluorescence staining was performed. Anti-insulin, anti-glucagon and anti-vp1 reactivities were detected using an Alexa Fluor 488-conjugated anti-guinea pig antibody, an Alexa Fluor 488-conjugated anti-rabbit antibody and an Alexa Fluor 568conjugated anti-mouse antibody, respectively (Invitrogen, Paisley, UK). Sections were mounted using Vectashield

Table 1 Analysis of islet staining with three anti-enteroviral antisera in 11 cases of type 1 diabetes

\begin{tabular}{cccccc}
\hline \multirow{2}{*}{ Number of ICIs } & \multicolumn{5}{l}{ Number of ICIs stained by: } \\
\cline { 2 - 6 } & $\begin{array}{l}\text { Any } \\
\text { antiserum }\end{array}$ & $\begin{array}{l}\text { Any two } \\
\text { antisera }\end{array}$ & $\begin{array}{l}\text { Dako } \\
5 \text { D } 8 / 1\end{array}$ & Kdf & $9 D 5$ \\
\hline 50 & $12(24 \%)$ & 5 & 7 & 0 & 10 \\
31 & $5(16 \%)$ & 0 & 3 & 2 & ND \\
18 & $3(17 \%)$ & 0 & 3 & 0 & ND \\
31 & $9(29 \%)$ & 5 & 7 & 7 & ND \\
28 & $4(14 \%)$ & 0 & 4 & 0 & 0 \\
21 & $21(100 \%)$ & $19^{a}$ & 18 & 20 & 19 \\
2 & $2(100 \%)$ & 0 & 2 & 0 & ND \\
26 & $18(69 \%)$ & 0 & 18 & 0 & ND \\
36 & $12(33 \%)$ & 0 & 12 & 0 & ND \\
23 & $3(13 \%)$ & 0 & 3 & 0 & ND \\
16 & $11(69 \%)$ & 0 & 11 & 0 & 0 \\
\hline
\end{tabular}

${ }^{\mathrm{a}}$ Eighteen islets stained by three antisera

$\mathrm{ND}$, not determined 
medium (Vector Labs, Burlingame, CA, USA) and were observed using a Zeiss LSM510 Meta confocal microscope (Welwyn Garden City, UK).

Statistics Results are shown as means \pm SEM. Statistical analysis was performed either by a $\chi^{2}$ test or Student's $t$ test, as appropriate.

\section{Results}

$v p 1$ staining in the islets of recent-onset type 1 diabetic patients In order to confirm the ability of the Dako enteroviral antibody used previously to identify vp1 immunoreactivity in the pancreas [13, 19] and intestine [20] of patients with type 1 diabetes, we initially examined its reactivity in formalin-fixed, paraffin-embedded heart and pancreas samples taken from five neonates having fulminant, fatal CVB infection. Cardiac myocytes and many pancreatic islets from these samples contained cells which were strongly immunopositive for vp1 (Fig. 1a and 3a). By contrast, none of the islets examined in normal pancreas sections recovered from 11 non-diabetic neonates, nor the myocytes in hearts of neonates without myocarditis, were immunopositive for vp1.

Probing of the pancreases from our cohort of type 1 diabetes patients with the Dako anti-vp1 antibody revealed the presence of small numbers of intensely stained cells in multiple islets from the majority of cases (Fig. 1b, c). These immunopositive islet cells were readily distinguishable from the surrounding unstained endocrine cells (which formed the majority in all cases), suggesting that the antibody did not label an endogenous islet cell antigen. To facilitate quantitative analysis, a case was deemed positive for $\mathrm{vp} 1$ when at least one intensely stained endocrine cell was present in an islet within any given section. Using this criterion, 44 of the 72 (61\%) recent-onset type 1 diabetes cases were positive for enteroviral vp1 antigen (Fig. 1g) and most contained multiple immunopositive cells in a range of islets. Comparison of the age and sex of the patients failed to reveal any significant differences between the vp1-positive and vp1-negative cases. However, the duration of diabetes tended to be lower in the vp1-positive group ( $2.32 \pm 0.8$ months vs $16.5 \pm 8.0$ months; $p=0.06$ ).

Occasional islet cell vp1 immunopositivity was detected in the pancreases of three of 39 (7.7\%) non-diabetic paediatric cases (Fig. 1e, g) but this amounted to a total of only four endocrine cells among the entire islet population examined. This was significantly lower than that observed in the patients with type 1 diabetes $\left(\chi^{2}=29.71, p<0.001\right)$. A series of 11 paediatric cystic fibrosis pancreases was also examined for $\mathrm{vp} 1$ immunopositivity to determine whether

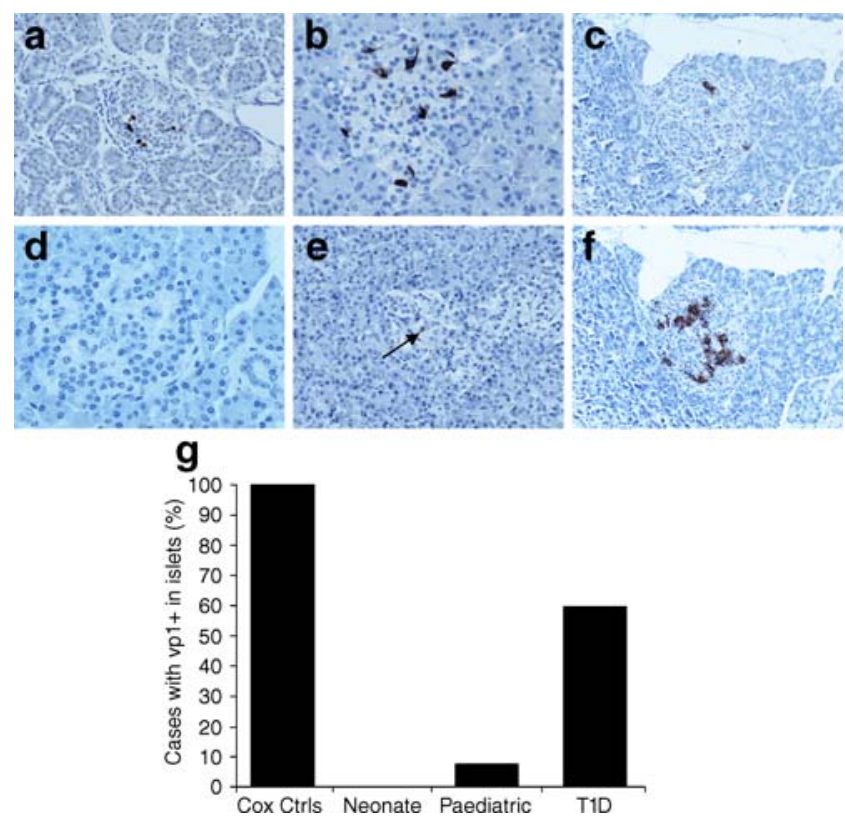

Fig. 1 Immunohistochemical analysis of vp1 staining in human pancreatic islets. Pancreas sections from a Coxsackie-infected neonatal child (a) and two different recent-onset type 1 diabetic patients $(\mathbf{b}, \mathbf{c})$ were stained for vp1. A serial section of the islet in (c) was also immunostained for insulin (f) and shows that most beta cells do not produce vp1. vp1 immunoreactivity was not observed in islets from the majority of paediatric cases (d). Only four positively stained islet cells were observed in three of 39 paediatric cases, one such cell is shown by an arrow (e). g The percentage of cases from each case group that were found to have at least one intensely stained cell (vp1+) within an islet. The number of positive cases/total number were five of five for Coxsackie-infected neonatal controls (Cox Ctrls), none of 11 for the normal neonates, three of 39 paediatric cases, and 44 of 72 type 1 diabetes cases (T1D)

the viral protein is found commonly in patients with another pancreatic inflammatory disease. Of these, two contained multiple vp1-positive islets similar to those seen in the type 1 diabetes patients (not shown). Importantly, subsequent examination of the case histories of these patients revealed that the two with vp1 immunopositivity in the islets also had diabetes. None of the vp1-negative cystic fibrosis cases had diabetes.

vp1 immunostaining is restricted to insulin-containing islets (ICIs) In the pancreas in recent-onset type 1 diabetes, approximately $60 \%$ of the islets are insulin deficient and the remainder contain residual insulin-secreting beta cells [18]. An analysis was made of the distribution of vp1-positive cells between insulin-deficient islets (IDIs) and ICIs in serial sections from ten patients with frequent vp1-positive endocrine cells. A total of 342 ICIs were analysed and 269 (78.7\%) were found to be positive for vp1. By contrast, only 16 of $611(2.6 \%)$ IDIs showed evidence of vp1 within the plane of the section (Fig. 2a). This suggests that beta cells contain the viral protein and confirmation was 

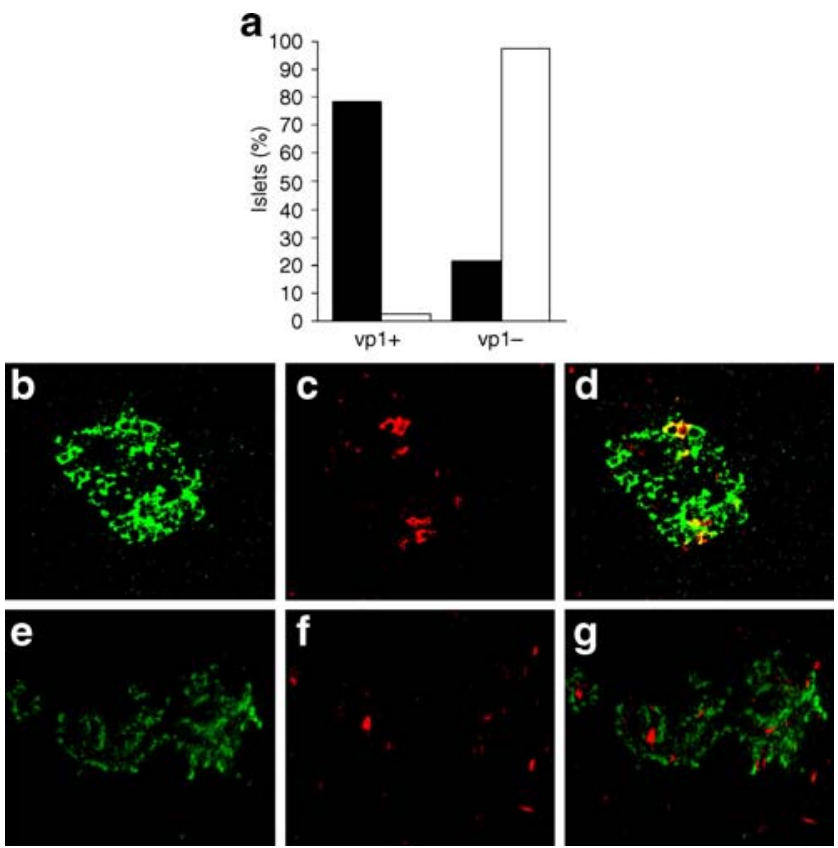

Fig. 2 Analysis of serial sections stained for vp1, insulin and glucagon. a vp1 production is restricted to insulin-containing beta cells. The percentage of ICIs (black bars) and IDIs (white bars) that stained positively for $\mathrm{vp} 1(\mathrm{vp}+)$ was determined. The results presented are from ten recent-onset type 1 diabetic cases who had vp1-positive endocrine cells; a total of 342 ICIs and 611 IDIs were analysed. Of these 269 and 16, respectively, were vp1-positive. b-g Confocal microscopic analysis of representative islets reveals that vp1 (red; $\mathbf{c}, \mathbf{d}$, f, g) co-localised with insulin (green; panels b, d) but not with glucagon $(\mathbf{e}, \mathbf{g})$. Double-positive cells are stained in yellow and are visible in $\mathbf{d}$ but not in $\mathbf{g}$

achieved more directly by double immunofluorescence staining for either insulin or glucagon together with vp1 (Fig. 2b-g). This revealed that staining of the viral antigen was restricted to a minority of insulin-positive cells. Similar results were verified in five separate cases, in which an average of 32 islets were examined per section.

The presence of $\mathrm{CD}_{4} 5^{+}$immune cells was also examined to determine whether a relationship existed between vp1 immunopositivity and the extent of insulitis in type 1 diabetes patients. A total of $200 \mathrm{vp} 1$-positive ICIs were studied (from seven patients) and 123 (61.5\%) of these showed no evidence of inflammation (i.e. they were negative for CD45).

Verification of islet vp1 staining with other enteroviral antisera To confirm that the Dako antiserum was detecting enteroviral $\mathrm{vp} 1$ in the islets of type 1 diabetes patients, the staining pattern was compared with that obtained using a second, polyclonal antiserum raised against vp1 (designated $\mathrm{Kdf}$ ), and an additional pan-enteroviral monoclonal antibody, not specifically directed against vp1 (clone 9D5). Initially these antisera were tested in normal and Coxsackieinfected neonatal human heart. This confirmed that similar regions of the tissue were stained with each antibody in

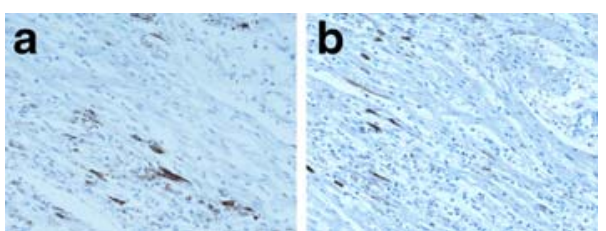

Fig. 3 Comparison of the staining patterns obtained in Coxsackieinfected neonatal heart with two antisera raised against vp1: Dako vp1 5D8/1 antibody (a) or the Kdf vp1 antibody (b)

infected tissue (examples for Dako and Kdf are shown in Fig. 3) and that staining was absent from normal control hearts. Under optimal conditions, the Dako antiserum appeared to be the most sensitive of the three, since staining was more intense and extensive with this antibody. It also gave more consistent results on tissues fixed in a variety of fixatives.

Comparison of staining with the three antisera applied to serial sections of pancreases is presented in Fig. $4 \mathrm{a}-\mathrm{f}$ together with a quantitative analysis from 11 patients (Table 1). The images show islets from two different patients stained with either the Dako vp1 (Fig. 4a, e), Kdf (Fig. 4b, f) or 9D5 (Fig. 4c) antibodies and demonstrate that intensely positive islet cells could be detected in the same islets with these reagents. Of the 11 patients studied in detail, one Bouin'sfixed case had islets stained by all three antisera and at least a further three had positive immunostaining in ICIs with two antibodies. The Dako antiserum appeared most sensitive, since it stained one or more of the ICIs in all 11 cases. No IDIs were stained with any of the antisera.

Correlation between islet vpl staining and additional markers of viral infection Serial sections of pancreases were next stained with antibodies to insulin, vp1, PKR and MHC-I in six vp1-positive type 1 diabetes patients to determine whether vp1 staining correlated with other markers of viral infection and autoimmunity. Representative ICIs from one of these patients are shown in Fig. 5a and a
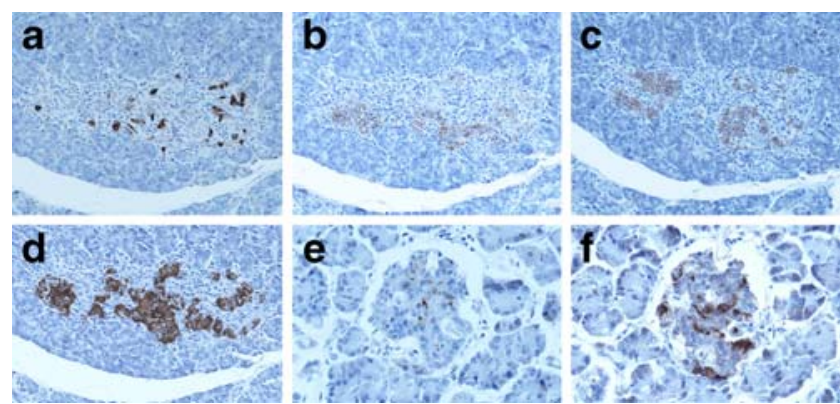

Fig. 4 Comparison of the staining patterns obtained in human type 1 diabetic pancreases with three different antisera raised against enteroviral antigens. Representative islets from two different type 1 diabetic pancreases (a-d and $\mathbf{e}, \mathbf{f})$ were stained with Dako vp1 antibody (a, e), Kdf vp1 (b, f), the anti-enteroviral antibody 9D5 (c) and insulin (d) 
Fig. 5 Examination of vp1 immunopositivity, production of PKR and hyperexpression of MHC class I in the islets of recent-onset type 1 diabetic patients and a patient with type 2 diabetes. a-c Two representative islets from recent-onset type 1 diabetic patients and one from a patient with type 2 diabetes were stained for insulin, vp1, PKR and MHC class I (MHCI). The panels show an ICI (a) and an IDI (b) from a patient with type 1 diabetes (T1D), and an islet from a type 2 diabetic patient (T2D) (c). Note in (c) that the same cells within the islet stain with vp1 and PKR on these adjacent sections. d Detailed analysis of 177 ICIs from six representative vp1-positive type 1 patients demonstrates that $\mathrm{vp} 1$ is associated with PKR production in $154(87 \%)$ of these ICIs. The number of ICIs vp1- PKR+, vp1+ PKR- and vp1- PKRwas seven, five and 11 , respectively

\section{a}

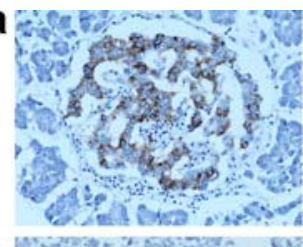

b

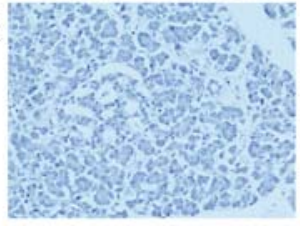

C

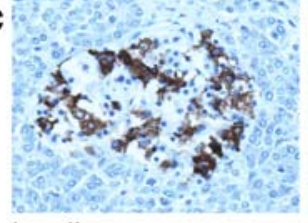

Insulin

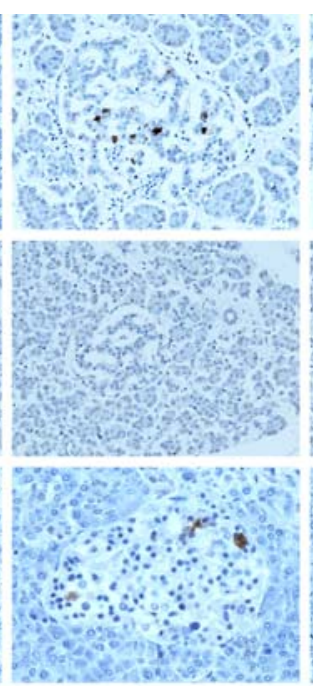

vp1

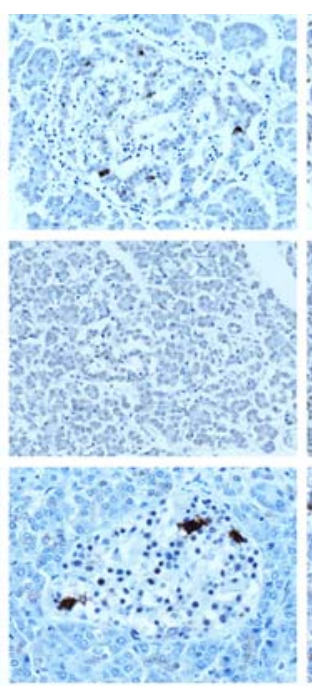

PKR

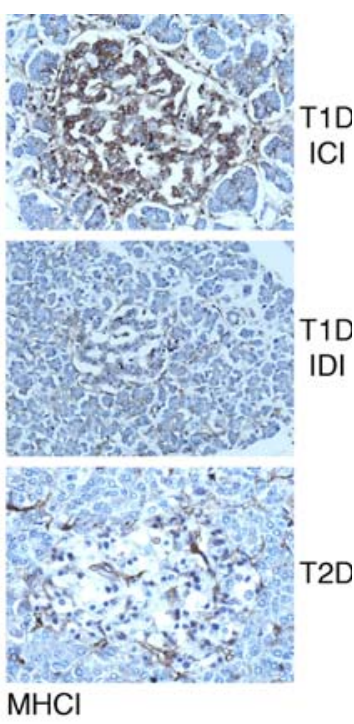

$1 \mathrm{D}$ $2 \mathrm{D}$

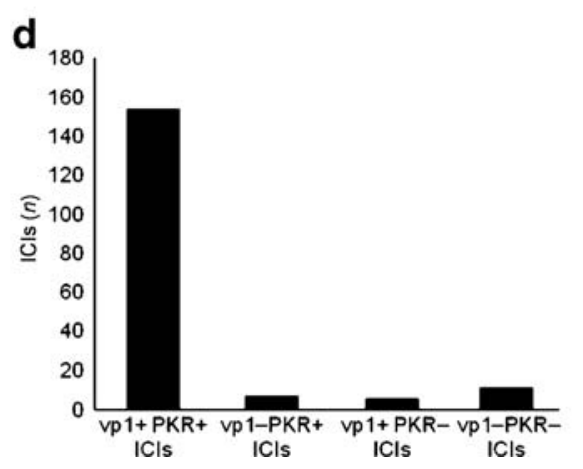

representative IDI is shown in Fig. $5 b$ for comparison. PKR staining correlated with vp1 immunopositivity in 154 of $177(87.0 \%)$ ICIs analysed in the type 1 diabetes patients (Fig. 5a, b), whereas it was not detected in any islets of five non-diabetic age-matched control pancreases. PKR staining was only rarely observed in IDIs. Islet cell MHC-I hyperexpression was detected in all of the type 1 diabetes cases analysed and this correlated with the presence of insulin (Fig. 5a). All vp1-positive islets were found to hyperexpress MHC-I, but so did many vp1-negative ICIs. MHC-I was not hyperexpressed in any neonatal or paediatric control pancreas.

$v p 1$ staining in sections of pancreases from type 2 diabetic patients and from non-diabetic adults The study was extended to include pancreas samples recovered post mortem from 69 normal adults and 25 patients with type 2 diabetes. Islet endocrine cell immunopositivity for $\mathrm{vp} 1$ (Dako antiserum) was observed in nine of 69 (13.0\%) normal pancreases (Fig. 6a and e). Of 25 type 2 diabetic patients analysed, ten $(40 \%)$ contained a range of islets having intensely positive cells (Figs $5 \mathrm{c}, 6 \mathrm{~b}$ and e; $\chi^{2}=7.89$, $p<0.01$ vs normal pancreas).
A more detailed comparison of the frequency of vp1positive islets in ten type 1 and six type 2 diabetic pancreases as well as seven non-diabetic adult pancreases was performed (Fig. 6f). The cases were chosen at random among pancreases which had vp1-positive islets. Seventy of 251 (27.9\%) islets contained vp1-positive endocrine cells in the six type 2 diabetes pancreas sections studied, whereas only 15 of 210 (7.1\%) islets from seven normal adult pancreases contained equivalent cells (Fig. 6f; $\chi^{2}=32.81, p<0.001$ ). This compared with 269 of $342(78.7 \%)$ vp1-positive ICIs in the ten type 1 diabetes cases studied in detail $\left(\chi^{2}=152.31, p<0.001\right.$; vs type 2 diabetes). In the islets of patients with type 2 diabetes islets that were vp1-positive, PKR immunopositivity was also often detected (Figs $5 \mathrm{c}$ and $6 \mathrm{~g}$ ). Hyperexpression of MHC-I was not seen in any islet from type 2 diabetes patients (Fig. 5c), nor in non-diabetic adult pancreases.

Finally, in some cases, focal pancreatic centroacinar cells in the exocrine tissue and some smooth muscle cells in vein walls were stained positively by the Dako anti-vp1 serum (Fig. 6c, d). Such staining was also seen, but much less frequently, in the neonatal and paediatric pancreases of both diabetic and non-diabetic patients, and it was never observed with the Kdf antiserum. 

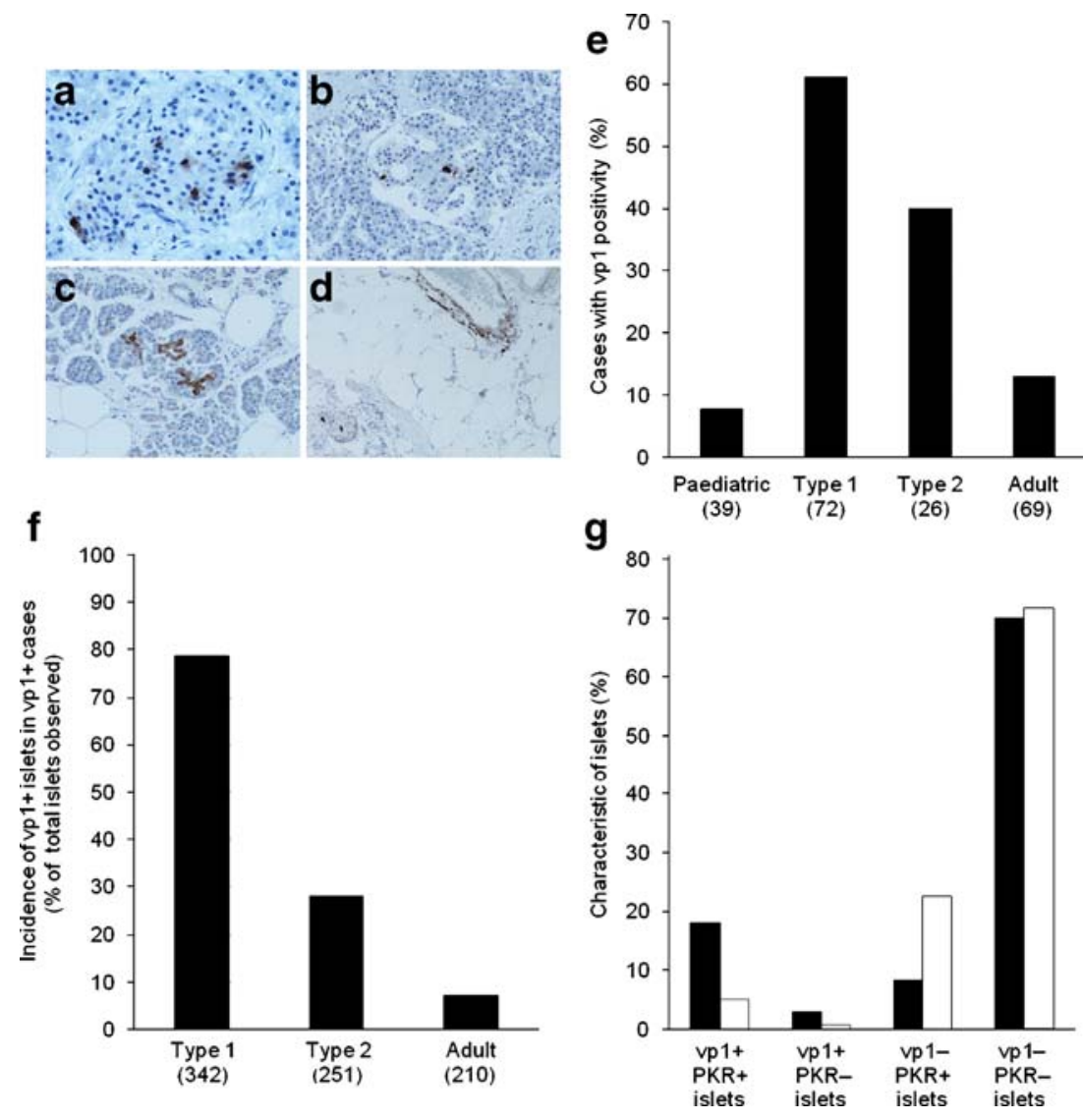

Fig. 6 a-d Immunostaining of vp1 in pancreas sections from patients with type 2 diabetes. Representative islets from a normal adult (a) or a patient with type 2 diabetes (b) were stained for vp1. In these cases, centroacinar (c) and smooth muscle (d) cell staining was also observed. e Analysis of the percentage of cases from each case group that have at least one intensely stained cell within an islet. The number of cases examined is shown in parentheses. $\mathbf{f}$ Detailed analysis of the

frequency of vp1 immunopositivity within ICIs in patients with type 1 diabetes $(n=10)$, type 2 diabetes $(n=6)$ and normal adults $(n=7)$. The number of islets studied in each category is shown in brackets. g Detailed analysis of PKR and vp1 production in up to 50 randomly selected ICIs from nine type 2 diabetic patients (339 islets; black bars) and nine normal adult (403 islets; white bars)

\section{Discussion}

Circumstantial evidence has implicated enteroviral infection in the aetiology of type 1 diabetes but direct support for the hypothesis that islet cell enterovirus infection is a common occurrence in the disease has been more difficult to obtain [1]. However, it was recently reported that vp1 was detectable in a range of beta cells in two of five recentonset type 1 diabetic patients and in another diabetic patient who had received a pancreatic transplant, but not in 26 nondiabetic controls [13]. In order to verify and extend this finding, we have analysed the prevalence of pancreatic islet vp1 immunopositivity in 72 recent-onset type 1 diabetic patients and in a range of controls. The results reveal a prevalence of islet $\mathrm{vp} 1$ immunopositivity of $\sim 61 \%$ in type 1 diabetic patients, with almost no equivalent staining in the pancreases of neonatal and paediatric non-diabetic controls. Double immunofluorescence studies showed that vp1 staining was confined to beta cells. Thus, the proposal that

vp1 immunopositivity may be common in the insulinsecreting cells of type 1 diabetic patients is supported by this more extensive analysis.

An important consideration is the identity of the antigen labelled by the Dako vp1 antiserum. Previously, the specificity of the Dako vp1 antiserum has been assessed by comparing immunohistochemical and enteroviral RNA detection techniques. For example, in a study of heart biopsies of 70 patients with dilated cardiomyopathy, enteroviral RT-PCR testing was positive in 25 samples. Seventeen of these had focal staining of cardiac myocytes by Dako vp1 antiserum, while all of the RT-PCR-negative samples were vp1-negative on immunohistochemistry. All 45 normal control hearts were negative with both techniques [21]. Additionally, RT-PCR for enterovirus was positive in salivary gland biopsies in seven of eight patients with primary Sjogren's syndrome but not in 16 normal salivary glands. Ductal, acinar and infiltrating inflammatory cells stained positively with Dako anti-vp1 in the Sjogren's syndrome cases but not in the controls [22]. 
We verified that the Dako vp1 antibody labelled human cardiac myocytes in neonates infected with a number of different CVB in vivo, whereas non-infected neonatal heart was immunonegative. However, in common with others $[19,23]$ we also noted that the Dako antiserum can, under certain conditions, label tissues in which vp1 production is not expected. In particular, our preliminary work revealed that, when applied to formalin-fixed, paraffin-embedded sections of Coxsackie-infected neonatal mouse organs, this antibody stained some tissues which were reported to be negative for viral RNA by in situ hybridisation [24]. These included smooth muscle, bone, dental and hair follicle epithelium, pancreatic duct and centroacinar cells. Moreover, these tissues were also stained by the antibody in uninfected neonatal mice. Thus, while it is clear that the Dako antibody is a sensitive agent for labelling enteroviral $\mathrm{vp} 1$ in a range of tissues in mouse and man, it is also evident that it can label non-infected cells, including, in the pancreas, vascular smooth muscle and centroacinar cells. However, it should be emphasised that in uninfected neonatal mice and neonatal children the antibody did not stain pancreatic endocrine cells. Thus, in the context of islets of Langerhans, the Dako antibody stained endocrine cells only in control tissues known to be infected by virus.

Polyclonal antisera raised against enteroviruses have been shown to cross react with heat shock protein (hsp)-60 [25] and the tyrosine phosphatase, IA-2 [26]. However, it seems unlikely that the Dako vp-1 antiserum recognised either of these proteins in the beta cells in this study. IA-2 is present in alpha cells as well as beta cells, while the vp1 staining was seen in a minority of beta cells, but not alpha cells. The expression of hsp-60 has been studied in these diabetic samples before [27] and there was no equivalent staining of occasional beta cells as described here for vp1.

Because of the potential for non-specific immunoreactions with the Dako antiserum and possible doubts about the origin of the virus isolated previously $[13,28]$ we used two additional antisera raised against enteroviral proteins to analyse the presence of enterovirus in sections of pancreas. With each of these antisera, specific enteroviral immunopositivity was first demonstrated in infected neonatal human hearts. Both antisera stained endocrine cells in ICIs, which correlated with immunopositivity detected with Dako anti-vp1 in some, but not all, type 1 diabetic patients tested.

In an earlier study [13] two additional techniques were used to verify that vp1 immunostaining in diabetic islets represented virus infection. First, virus particles were observed by electron microscopy, confined to beta cells, in the same cases as had demonstrable vp1. Second, a strain of CVB4 was isolated from one of the pancreas samples and shown to be capable of infecting normal human islets in vitro, leading to impaired glucose-stimulated insulin secretion [13]. While this evidence appears persuasive, others [28] have cast doubt on the origin of the virus strain, by suggesting that the reported RNA sequence analysis implies possible contamination with a laboratory strain.

Since most of the previous studies [13, 21, 22] were conducted using fresh biopsy tissues, or tissues removed at the time of organ donation, the authors were able to employ electron microscopy, RT-PCR or in situ hybridisation techniques to confirm the validity of the vp1 staining. These options were not open to us since the pancreatic material from patients with diabetes in the present study was collected almost entirely retrospectively from autopsies performed hours after the death of patients. In previous work [29] attempts were made to detect enteroviral RNA by in situ hybridisation in this collection and no evidence of infection was found. The poor quality of RNA in formalinfixed, paraffin-embedded autopsy pancreas, probably the result of early RNA degradation in a tissue rich in RNAse, was exemplified in that study by the fact that mRNA for insulin could be detected by in situ hybridisation in only 29 of the 75 pancreases studied, even though all had insulincontaining beta cells as shown by immunohistochemistry. In support of this, others also failed to detect viral RNA by in situ hybridisation in an autopsy pancreas where evidence of viral protein production was detected by immunohistochemistry [19]. Thus no attempt was made in the present study to repeat the search for enteroviral RNA by in situ hybridisation or RT-PCR. Rather, we looked at the production of another protein that is upregulated in response to enteroviral infection, PKR [30], and found a strong concordance between PKR immunopositivity and that for $\mathrm{vp} 1$ in the islets of both type 1 and type 2 diabetic patients. This is arguably the best evidence in the present study that detection of vp1 by the Dako antibody in beta cells is indicative of a persistent enteroviral infection.

While staining of vp1 correlated with PKR immunostaining in the islets of patients with type 1 diabetes it was not seen in many of the ICIs where there was hyperexpression of class I MHC on endocrine cells. This latter phenomenon is intimately associated with IFN- $\alpha$ production by beta cells [17] and is unique to type 1 diabetes [31]. Thus, vp1 staining of beta cells does not appear to be directly associated with secretion of IFN- $\alpha$ and it is unclear why IFN- $\alpha$ is produced by beta cells in those islets which hyperexpress class I MHC [26] but have no demonstrable viral capsid protein.

In experimental acute enteroviral myocarditis in mice, viral capsid protein was most easily detectable by immunohistochemistry under conditions where there was an excess of positive- over negative-strand viral RNA within infected cells. By contrast, when the infection continued into a chronic phase, the number of positive and negative enteroviral RNA strands per cell became equivalent and capsid 
protein was less readily demonstrable [32]. On this basis, it is conceivable that a molecular form of virus that is not readily detectable by immunohistochemistry for vp1, may exist as a chronic infection in many of the beta cells in type 1 diabetes and that, occasionally, production of the whole virus increases, leading to capsid protein synthesis in a small number of beta cells. If this hypothesis is correct, then our results imply that the molecular form of virus detectable by vp 1 immunostaining is also the form that most potently induces PKR production, whereas IFN- $\alpha$ may be induced by both molecular forms.

Extending the current analysis revealed that, surprisingly, $40 \%$ of adult type 2 diabetes cases displayed focal staining for $\mathrm{vp} 1$ in their islets. The staining pattern was indistinguishable from that seen in the islets of patients with type 1 diabetes (although it occurred much less frequently in type 2 diabetes), which might suggest that immunopositivity for vp1 occurs as a consequence of diabetes. Alternatively, enteroviral infection could play an important role in the pathology of beta cell loss and malfunction in both type 1 and type 2 diabetes.

The latter proposition is consistent with evidence that type 2 diabetes may be associated with islet cell inflammation [33], although it must also be noted that there is no induction of autoimmunity in type 2 diabetes. Nevertheless, our present finding of production of PKR in consort with vp1 in the islets of patients with type 2 diabetes is consistent with an active response by infected endocrine cells to the presence of virus in this disease. Since non-cytopathic infection of beta cells by enterovirus leads to decreased glucose-induced insulin secretion in vitro [13] it is conceivable that, in a person with obesity and increased insulin resistance, in whom the demand for insulin is raised, such an infection may contribute to beta cell dysfunction and the development of type 2 diabetes, even if not associated with autoimmunity. Our findings could also suggest a similar role for enterovirus infection in precipitating clinical diabetes in some patients with cystic fibrosis.

vp1 staining in islets was found in $\sim 10 \%$ of non-diabetic controls of all ages. This is consistent with studies showing that $4-5 \%$ of normal children had enteroviral RNA in their serum $[4,7,8]$ and $11.8 \%$ had enteroviral RNA present in faecal samples [34, 35]. Since a range of enteroviruses can infect human islets in vitro $[12,36,37]$ it is possible that the pancreas is frequently colonised during an enteroviral infection.

Overall, our results are consistent with the hypothesis that enteroviral infection of beta cells at a young age, in patients with a genetic predisposition to type 1 diabetes, might precipitate autoimmunity. The islet reaction to that infection would include production of PKR, secretion of IFN $-\alpha$ and abnormal production of MHC molecules, leading to the type 1 form of the disease. Infection by a similar virus, in a person with a different genetic background, might be associated with PKR production, but if it did not precipitate IFN- $\alpha$ production by beta cells, or abnormalities of islet MHC expression in islets, this infection would not be associated with autoimmunity, and in the great majority of patients would lead to no clinical disease. However, in patients with an already compromised metabolic situation, as a result of insulin resistance, it may contribute to the development of type 2 diabetes. Further studies, including viral RNA detection techniques on fresh, organ-donation, pancreas samples from patients with recent-onset disease, are indicated to confirm the presence and identity of enterovirus strains causing diabetes.

Acknowledgements We thank Diabetes UK, the Juvenile Diabetes Research Foundation and a Coordinated Action of the European Union (TONECA) for financial support of this study. We also thank R. Kandolf (Tübingen, Germany) and J. Neefjes (Amsterdam) for their kind gifts of vp1 and MHC-I antibodies used in this work and D. Hilton (Derriford Hospital, Plymouth, UK) for paraffin-embedded Coxsackie-infected neonatal mouse sections.

Duality of interest The authors declare that there is no duality of interest associated with this manuscript.

\section{References}

1. Gale EAM (2008) Congenital rubella: citation virus or viral cause of type 1 diabetes? Diabetologia 51:1559-1566

2. Gamble DR, Kinsley ML, FitzGerald MG, Bolton R, Taylor KW (1969) Viral antibodies in diabetes mellitus. BMJ 3:627-630

3. Andreoletti L, Hober D, Hober-Vandenberghe C et al (1997) Detection of Coxsackie B virus RNA sequences in whole blood samples from adult patients at the onset of type I diabetes mellitus. J Med Virol 52:121-127

4. Clements GB, Galbraith DN, Taylor KW (1995) Coxsackie B virus infection and onset of childhood diabetes. Lancet 346:221223

5. Coutant R, Carel JC, Lebon P, Bougneres PF, Palmer P, CanteroAguilar L (2002) Detection of enterovirus RNA sequences in serum samples from autoantibody-positive subjects at risk for diabetes. Diabet Med 19:968-969

6. Elfaitouri A, Berg AK, Frisk G, Yin H, Tuvemo T, Blomberg J (2007) Recent enterovirus infection in type 1 diabetes: evidence with a novel IgM method. J Med Virol 79:1861-1867

7. Moya-Suri V, Schlosser M, Zimmermann K, Rjasanowski I, Gurtler L, Mentel R (2005) Enterovirus RNA sequences in sera of schoolchildren in the general population and their association with type 1-diabetes-associated autoantibodies. J Med Microbiol 54:879-883

8. Nairn C, Galbraith DN, Taylor KW, Clements GB (1999) Enterovirus variants in the serum of children at the onset of Type 1 diabetes mellitus. Diabet Med 16:509-513

9. von Herrath M (2009) Can we learn from viruses how to prevent type 1 diabetes? The role of virus infections in the pathogenesis of type 1 diabetes and the development of novel combination therapies. Diabetes 58:2-11

10. Sarmiento L, Cabrera-Rode E, Lekuleni L et al (2007) Occurrence of enterovirus RNA in serum of children with newly diagnosed type 1 diabetes and islet cell autoantibody-positive subjects in a 
population with a low incidence of type 1 diabetes. Autoimmunity 40:540-545

11. Yoon JW, Austin M, Onodera T, Notkins AL (1979) Isolation of a virus from the pancreas of a child with diabetic ketoacidosis. $\mathrm{N}$ Engl J Med 300:1173-1179

12. Ylipaasto $P$, Klingel K, Lindberg AM et al (2004) Enterovirus infection in human pancreatic islet cells, islet tropism in vivo and receptor involvement in cultured islet beta cells. Diabetologia 47:225-239

13. Dotta F, Censini S, van Halteren AG et al (2007) Coxsackie B4 virus infection of beta cells and natural killer cell insulitis in recent-onset type 1 diabetic patients. Proc Natl Acad Sci U S A 104:5115-5120

14. Tam PE, Messner RP (1999) Molecular mechanisms of Coxsackie virus persistence in chronic inflammatory myopathy: viral RNA persists through formation of a double-stranded complex without associated genomic mutations or evolution. J Virol 73:1011310121

15. Ylipaasto P, Kutlu B, Rasilainen S et al (2005) Global profiling of Coxsackie virus- and cytokine-induced gene expression in human pancreatic islets. Diabetologia 48:1510-1522

16. Chehadeh W, Kerr-Conte J, Pattou F et al (2000) Persistent infection of human pancreatic islets by Coxsackie virus $B$ is associated with alpha-interferon synthesis by beta-cells. J Virol 74:10153-10164

17. Foulis AK, Farquharson MA, Meager A (1987) Immunoreactive alpha-interferon in insulin-secreting beta cells in type 1 diabetes mellitus. Lancet 2:1423-1427

18. Foulis AK, Liddle CN, Farquharson MA et al (1986) The histopathology of the pancreas in type 1 (insulin-dependent) diabetes mellitus: a 25 -year review of deaths in patients under 20 years of age in the United Kingdom. Diabetologia 29:267-274

19. Oikarinen M, Tauriainen S, Honkanen T et al (2008) Analysis of pancreas tissue in a child positive for islet cell antibodies. Diabetologia 51:1796-1802

20. Oikarinen M, Tauriainen S, Honkanen T et al (2008) Detection of enteroviruses in the intestine of type 1 diabetic patients. Clin Exp Immunol 151:71-75

21. Andréoletti L, Bourlet T, Moukassa D et al (2000) Enteroviruses can persist with or without active viral replication in cardiac tissue of patients with end-stage ischemic or dilated cardiomyopathy. J Infect Dis 182:1222-1227

22. Triantafyllopoulou A, Tapinos N, Moutsopoulos HM (2004) Evidence for coxsackievirus infection in primary Sjögren's syndrome. Arthritis Rheum 50:2897-2902

23. Klingel K, Sauter M, Bock CT, Szalay G, Schnorr JJ, Kandolf R (2004) Molecular pathology of inflammatory cardiomyopathy. Medical Microbiol Immunol 193:101-107
24. Hilton DA, Day C, Pringle JH, Fletcher A, Chambers S (1992) Demonstration of the distribution of coxsackie virus RNA in neonatal mice by non-isotopic in situ hybridization. J Virol Methods 40:155-162

25. Härkönen T, Puolakkainen M, Sarvas M, Airaksinen U, Hovi T, Roivainen M (2000) Picornavirus proteins share antigenic determinants with heat shock proteins 60/65. J Med Virol 62:383-391

26. Härkönen T, Lankinen H, Davydova B, Hovi T, Roivainen M (2002) Enterovirus infection can induce immune responses that cross-react with beta-cell autoantigen tyrosine phosphatase IA-2/ IAR. J Med Virol 66:340-350

27. Mallard K, Jones DB, Richmond J, McGill M, Foulis AK (1996) Expression of the human heat shock protein 60 in thyroid, pancreatic, hepatic and adrenal autoimmunity. J Autoimmun 9:89-96

28. Drescher KM, Tracy SM (2008) The CVB and etiology of type 1 diabetes. Curr Top Microbiol Immunol 323:259-274

29. Foulis AK, McGill M, Farquharson MA, Hilton DA (1997) A search for evidence of viral infection in pancreases of newly diagnosed patients with IDDM. Diabetologia 40:53-61

30. Everett H, McFadden G (1999) Apoptosis: an innate immune response to virus infection. Trends Microbiol 7:160-165

31. Foulis AK, Farquharson MA, Hardman R (1987) Aberrant expression of class II major histocompatibility complex molecules by B cells and hyperexpression of class I major histocompatibility complex molecules by insulin containing islets in type 1 (insulindependent) diabetes mellitus. Diabetologia 30:333-343

32. Klingel KC, Hohenadl C, Canu A et al (1992) Ongoing enterovirus-induced myocarditis is associated with persistent heart muscle infection: quantitative analysis of virus replication, tissue damage and inflammation. Proc Natl Acad Sci U S A 89:314-318

33. Ehses JA, Perren A, Eppler E et al (2007) Increased number of islet-associated macrophages in type 2 diabetes. Diabetes 56 : 2356-2370

34. Witso E, Palacios G, Cinek O et al (2006) High prevalence of human enterovirus a infections in natural circulation of human enteroviruses. J Clin Microbiol 44:4095-4100

35. Cinek O, Witso E, Jeansson S et al (2006) Longitudinal observation of enterovirus and adenovirus in stool samples from Norwegian infants with the highest genetic risk of type 1 diabetes. $\mathrm{J}$ Clin Virol 35:33-40

36. Roivainen M (2006) Enteroviruses: new findings on the role of enteroviruses in type 1 diabetes. Int J Biochem Cell Biol 38:721725

37. Roivainen M, Knip M, Hyoty $\mathrm{H}$ et al (1998) Several different enterovirus serotypes can be associated with prediabetic autoimmune episodes and onset of overt IDDM. Childhood Diabetes in Finland (DiMe) Study Group. J Med Virol 56:74-78 